\title{
An automatic parameter extraction method for the 7x50m Stroke Efficiency Test
}

\author{
Marc Bächlin, Kilian Förster, Johannes Schumm, Dominik Breu, \\ Jürg Germann and Gerhard Tröster \\ Wearable Computing Laboratory, ETH Zürich, Zürich, Switzerland \\ E-mail: \{baechlin, foerster, troester\}aife.ee.ethz.ch
}

\begin{abstract}
We developed an automatic method to extract the parameters of the $7 \times 50 \mathrm{~m}$ Stroke Efficiency Test for swimming based on a wrist worn acceleration sensor device. In the wrist acceleration signal we detect characteristic swim events such as wall push-offs, wallstrikes and strokes. Based on this information we compute the distance per stroke and the swimming velocity. The upper error bounds of our automatic method are $1.67 \%$ for the velocity and $1.33 \%$ for the time per stroke. The velocity measurement accuracy is of comparable order to the manual accuracy. The automatic method clearly outperforms the manual measurement for the time per stroke extraction.
\end{abstract}

\section{Introduction}

The performance of swimming is strongly related to the swimmer's technique. Therefore a substantial proportion of the training of competitive swimmer is devoted to the improvement of the swimmer's style. To evaluate the improvement of the technique, the swimmer is interested in determining the efficiency of his strokes. The parameters to determine the efficiency of the stroke mechanics are the distance per stroke (stroke length) and the swimming speed [1].

It is common for professional swimmers to evaluate their swimming technique. The most accurate technique used today for gaining these parameters for professional swimmers is video analysis [2, 3]. The speed and the stroke length are extracted from a video recording of the swimmer. This is complex, time consuming and relies on an expensive swimming pool infrastructure. Recreational swimmers however do not have the knowledge and the methods to perform such tests.

There are also other methods, where the swimmer or a second person counts the number of strokes per lap and estimates the speed by measuring the time for one lap. This is either very inaccurate because the swimmer can not measure the exact time for one lap or time and labor consuming as each swimmer has to be observed independently by a second person (usually a trainer).

Pervasive computing has successfully been applied to different areas in the world of sports like football games [4, 5], skiing [6], running [7], table tennis [8] and rowing [9]. There have also been approaches in swimming to discriminate the stroke phase and quantify the swimmer's fatigue [10] or extract characteristic events for crawl swimming [11, 12].

However, an automated performance analysis in a typical swim technique evaluation test, such as the one we present in this paper, has not been investigated. In order to release trainers from time taking tasks and provide new evaluation techniques to recreational swimmers, we evaluate an automatic parameter extraction methodology. In our study we analyze the usability and accuracy of an automatic system to evaluate the swimmer's stroke efficiency. We developed a waterproof recording device based on a $3 \mathrm{D}$ acceleration sensor. We measure the wrist acceleration in freestyle swimming during the $7 \times 50 \mathrm{~m}$ Stroke Efficiency Test [1, 13]. In the wrist acceleration signal we detect characteristic events such as pool-starts, wall-strikes and strokes. Based on this information we compute the distance per stroke and the swimming velocity needed for the swimming technique analysis.

The remaining of the paper is structured as follows: In section 2 we provide background information on swim technique evaluation. Section 3 describes the device used and the experiment done for the evaluation of our method. In section 4 we describe our algorithms and evaluation methodology. In section 5 we present and discuss the results. Finally we conclude our work in section 6 and give an outlook on future research. 


\section{Swim technique evaluation}

Swimmers and their coaches want to know their progress in training. Dedicated swim tests are used to analyze the progress of the swimmer's technique and to ensure the training program has the right effect on the athlete. With an improved stroke technique, fewer strokes per lap are needed while swimming at constant speed. On the other hand, the swimmer's technique has improved if he can swim faster with the same number of strokes per lap [1]. This applies to all swimming styles. With increasing velocity and exhaustion "slippage" increases. This results in an increasing number of strokes per lap.

The most accurate technique nowadays for determining the distance per stroke is video recordings of the swimmer in a pool with special distance markers [2, 3].

A less accurate method to get a rough measure on the stroke efficiency without instrumentation is counting the number of strokes needed per lap. The number of strokes per lap is inaccurate because on the one hand the duration of traveling under water after the push-off varies, on the other hand the swimming speed is not taken into account. However, due to its simplicity this is still the most common technique, especially for nonprofessional swimmers.

An improved method to determine stroke rate, distance per stroke, and velocity without utilizing video recordings has been suggested by Maw and Volkers [13]. A second person measures manually three time intervals ignoring the start and the stop phase at the beginning and at the end of the lane. From the time measurements and the known mid-pool distance it is possible to calculate the velocity, the distance per stroke and the stroke rate. This method is described in detail below and acts as a reference for the further evaluation of our automated approach:

For the $7 \times 50 \mathrm{~m}$ Stroke Efficiency Test a $50 \mathrm{~m}$ long course pool is mandatory. Seven laps have to be swum with different speeds. The slowest lap is done approximately $12 \mathrm{sec}$ slower than the fastest lap. Each of the following sets is then undertaken approximately $2 \mathrm{sec}$ faster than the preceding one - the seventh and final one being the fastest with maximum effort. All sets are started with a push-off start in the pool. Between each run there is a recovery time of 2 minutes.

For each lap the time between the passes of the backstroke turn flags ( 5 and $45 \mathrm{~m}$ marks) is measured with a stopwatch - taking the swimmers head as the reference. At approximately the $10 \mathrm{~m}$ mark (respectively within the first $25 \mathrm{~m}$ segment) and at the $35 \mathrm{~m}$ mark (respectively within the second $25 \mathrm{~m}$ segment) the time needed for three complete stroke cycles is measured with a sec- ond stopwatch.

Ideally the swimmer has surfaced from his push-off start by the $5 \mathrm{~m}$ mark to ensure an accurate measurement. The velocity $(V)$ is calculated from the time needed for the $40 \mathrm{~m}$ mid-pool distance. The average time per stroke (TPS) is determined out of the time needed for the six measured strokes (three at the beginning and three at the end). The distance per stroke $(D P S)$ is finally calculated by

$$
\begin{gathered}
T P S=\frac{t_{3 \_s t r o k e s \_b}+t_{3 \_s t r o k e s \_e}}{6} \\
D P S=V \times T P S
\end{gathered}
$$

For explanation, three artificial examples of typical Stroke Efficiency Test graphs (DPS v.s. $V$ ) are illustrated in figure 1. Swimming technique gets better ei-

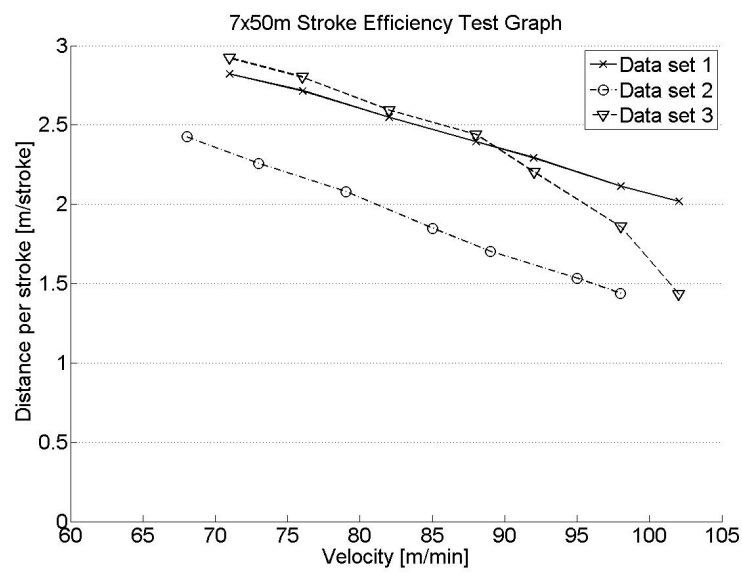

Figure 1. Three artificial examples of Stroke Efficiency Test graphs (DPS v.s. $V$ )

ther when the DPS increases with constant speed (the graph moves upwards, compare subject one with subject two in figure 1) or when speed increases with constant DPS (the graph moves to the right). It is desirable to maintain a good swimming stroke mechanics throughout the different swimming speeds. Better performing swimmers are able to 'hold their stroke together' at the fastest speeds resulting in a linear graph shown in data set one and two in figure 1. Less-skilled performers lose control of their technique, evidenced by a non-linear change in distance per stroke (see graph of third data set in figure 1). The inspection of the graph indicates the speed at which the control of the swim stroke starts to deteriorate. 


\section{Hardware and experiment}

For the usability analysis of the automatic parameter extraction in the Stroke Efficiency Test we accomplished a swim experiment according to the protocol described in section 2. For the experiment each swimmer has worn a SwimRecorder as depicted in figure 2.

The $S$ wimRecorder, derived from the device described in [14], consists of a 3-axes accelerometer, a microcontroller, $1 G B$ of flash memory (MultiMedia Card) and a rechargeable battery. The acceleration signals are A/D converted by a 10 Bit A/D converter and sampled at a frequency of $256 \mathrm{~Hz}$. With the $3.7 \mathrm{~V}$, $250 \mathrm{~mA}$ battery pack continuous data recording for up to 48 hours is possible. The fixation of the device to the swimmer is wristwatch alike. With a weight of only $34 \mathrm{~g}$ for the whole setup and the low profile of $12 \mathrm{~mm}$ the device is unobtrusive and not hindering while swimming.

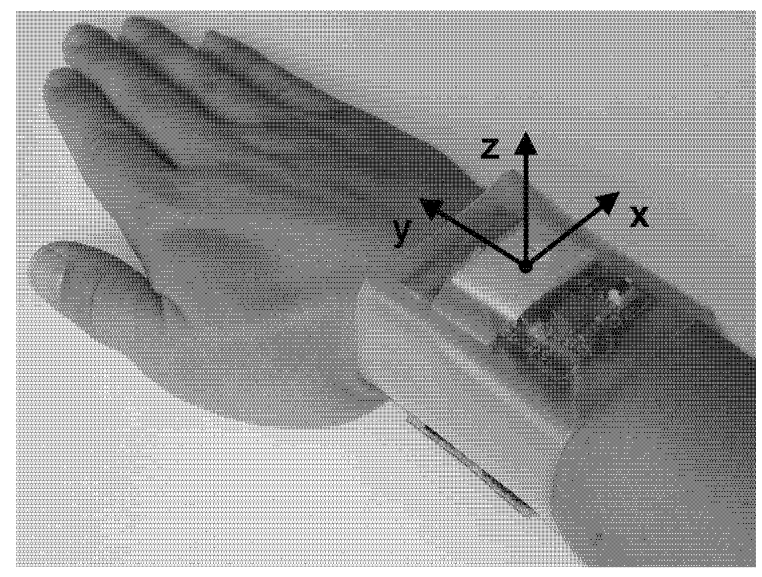

Figure 2. SwimRecorder attached to the swimmers wrist. The arrows indicate the orientation of the three axes of the acceleration sensor.

Each of the eight participants swam the $7 \times 50 \mathrm{~m}$ in crawl technique on a $2 \mathrm{~min}$ cycle with the $S$ wimRecorder attached to the right wrist (see figure 2). The swimmer holds on to the wall with his right hand and starts swimming on a signal from the experiment leader. The pool start is defined as the push-off from the pool wall. The end of the lap is defined as a wall-strike with the right hand. In addition to the wrist acceleration we measured manually the time intervals as explained in section 2. For comparison reasons the time at the $5 \mathrm{~m}$ mark, the $45 \mathrm{~m}$ mark and the end of the lap, as well as the times for three strokes at the beginning and at the end, were measured by an experimenter using a normal stopwatch. In addition to the stop watch times an-
Table 1. Label definitions

\begin{tabular}{|c|c|}
\hline Label & Defined moment \\
\hline \hline START $(0 \mathrm{M})$ & Hands are released from pool wall $0 \mathrm{~m}$ \\
\hline $5 \mathrm{M}$ & Head passes the $5 \mathrm{~m}$ mark \\
\hline STROKE & Right hand touches the water \\
\hline $45 \mathrm{M}$ & Head passes the $45 \mathrm{~m}$ mark \\
\hline END $(50 \mathrm{M})$ & Wall stroke at the end of lap $(50 \mathrm{~m})$ \\
\hline
\end{tabular}

other experimenter labeled the start and the end of the lap, the $5 \mathrm{~m}$ and $45 \mathrm{~m}$ passes as well as all strokes. For the labeling we used a hand-held PC running the CRN Toolbox [15]. The labeling was done by pressing the corresponding keys on the hand held PC. To ensure accurate and consistent labels the labeling moments were defined as listed in table 1 .

Eight subjects participated in this study, four from a competitive swimming club and 4 recreational swimmers. In total we recorded datasets of 56 laps. For each lap we have the acceleration data and the time stamps acquired by the stopwatch and the labeling device.

\section{Automatic parameter extraction method}

For the Stroke Efficiency Test graph the swimmer's distance per stroke is plotted versus the swimming velocity $[1,13]$. The parameter that need to be extracted from the acceleration data are therefore the swimmers velocity and the $D P S$.

At first a rough segmentation based on the mean of the raw $y$-acceleration signal is applied to localize the mid-pool area. The mean-value is calculated using a sliding window of $3.5 \mathrm{sec}$. Segment begin and end are detected by a drop in the mean value below $0.8 \mathrm{~g}$.

\subsection{Velocity extraction}

From the acceleration signal alone it is not possible to detect the swimmer's position in the pool without further constraints. Therefore the time for the whole $50 \mathrm{~m}$ of the pool is extracted by detecting the wall-push-off and the wall-strike events. This detection is based on the gradient of the y-axis. First the raw acceleration signal is low-pass filtered using a 2nd order Butterworth filter with a normalized cutoff frequency of 0.01 . The gradient is calculated with a sliding window of $1 / 5 \mathrm{sec}$ over the filtered signal.

The wall-push-off event is identified by first minimum in the gradient (first falling slope, see figure 3a). The wall-strike at the end of the pool is discriminated 
by the maximum in the gradient after the mid-pool area (increasing slope at the end, see figure $3 \mathrm{c}$ ).

\subsection{Distance per stroke extraction}

Extracting the DPS directly from the acceleration signal is not possible. With the velocity (section 4.1) and the TPS the DPS can be calculated according to equation 2.

To determine the TPS the timestamps of all stroke events are extracted from the acceleration data The most significant stroke-phase for the automatic stroke event detection is the moment when the hand is pulled upwards out of the water. This is represented as a negative peak in the y-axes of the acceleration signal. A minimum peak detection is applied to the butterworth filtered signal (see section 4.1) in the pre-segmented midpool area, marking all stroke occurrences (figure $3 \mathrm{~b}$ ).

With the timestamps of all stroke events the time needed for each stroke can be calculated. The TPS value for a specific lap is the average over all stroke time intervals within this lap.

\section{Results and discussion}

We first assess the reliability of the automatic parameter extraction algorithm. We compare the positions of the detected events to the positions we expect from the visual inspection of the data. The manual annotation serves as an aid to find the areas of interest in the datasets.

The start and the stop events are automatically detected in all laps of all subjects in the dataset. The deviation from the expected positions is in the range of $\pm 0.3 \mathrm{sec}$ for the pool-start and $\pm 0.2 \mathrm{sec}$ for the wallstrike. The strokes are detected reliably. Occasionally the first or the last stroke in one lap is missed. This is not critical as this does not affect the TPS. The maximum error of the detected stroke events from the expected positions is in the range of $\pm 10 \%$ of the TPS. This deviation depends mainly on the swimmers style and the swimming speed as this influences the width of the peak to detect. Figure 3 shows the acceleration signal of one example lap with the automatic detected strokes, wallpush off (start) and wall-strike (stop) events. From the errors of the automatic event detection we calculate the errors introduced to the velocity and the TPS calculation. Assuming a high velocity of $100 \mathrm{~m} / \mathrm{min}$ and a small number of 15 strokes per lap the upper bounds of the errors are $1.67 \%$ for the velocity and $1.33 \%$ for the TPS.

For comparison reason we also evaluate the accuracy of the manual measurements using the stopwatch and

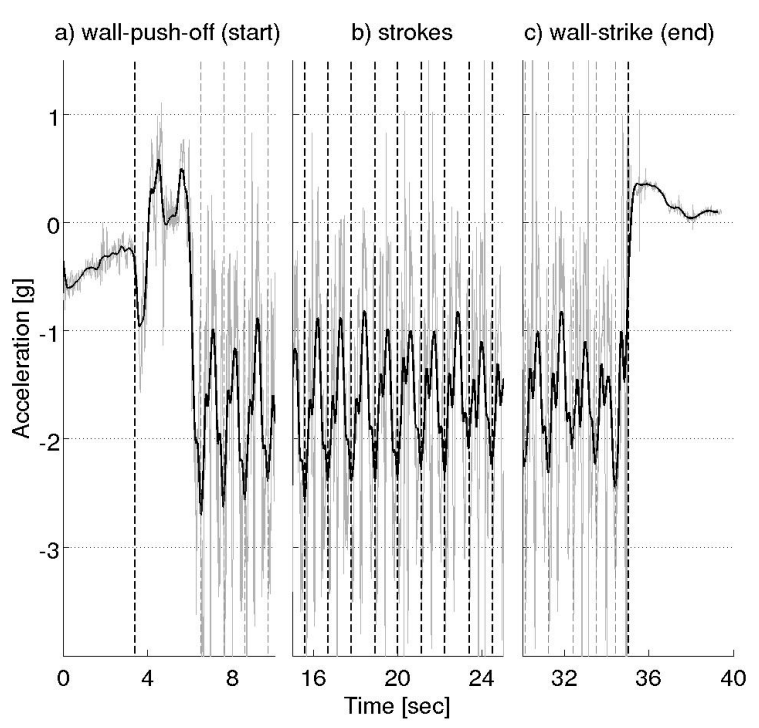

Figure 3. Detected wall-push-off, strokes and wall-strike events (gray: y-axis acceleration signal, black: filtered y-axis signal, dashed: detected events)

the labeling device. The comparison of the two manual measurements reveals an error with a standard deviation of $\pm 0.2 \mathrm{sec}$ per time event (start, stop, stroke). The manual time measurement errors are due to the human reaction time and to the variation in human event identification. Recalling the procedure from section 2 where the trainer measures different times to gain the velocity and the TPS of a swimmer, we can calculate the errors introduced to these parameters. Assuming a high swimming velocity of $100 \mathrm{~m} / \mathrm{min}$ and a low TPS of $1 \mathrm{sec}$, the upper bounds for these errors are $\pm 1.33 \%$ for the velocity and $\pm 13,3 \%$ for the TPS.

The manual velocity measurement is slightly more accurate than the automatic one. With the TPS extraction it is contrary, here the automatic method clearly outperforms the manual measurement. As described in section 4.1 the velocity calculation in the automatic method is based on the whole $50 \mathrm{~m}$ lap. This is in contrast to the manual method where only the $40 \mathrm{~m}$ midpool is used. We evaluate the influence of taking the full $50 \mathrm{~m}$ instead of only the mid-pool area. From the manual measurements we calculate the speed over the $40 \mathrm{~m}$ and the $50 \mathrm{~m}$ distance. In our dataset the velocity measured over $50 \mathrm{~m}$ is in average $2.5 \mathrm{~m} / \mathrm{min}$ faster then the velocity measured over $40 \mathrm{~m}$. This can be explained by the wall-push-off which causes a higher velocity in the first $5 \mathrm{~m}$ of the lap. Figure 4 shows two Stroke Efficiency Test graphs from the same subject. The first one is generated from the manual measurements using the $40 \mathrm{~m}$ 
mid-pool area for the velocity calculation. The second

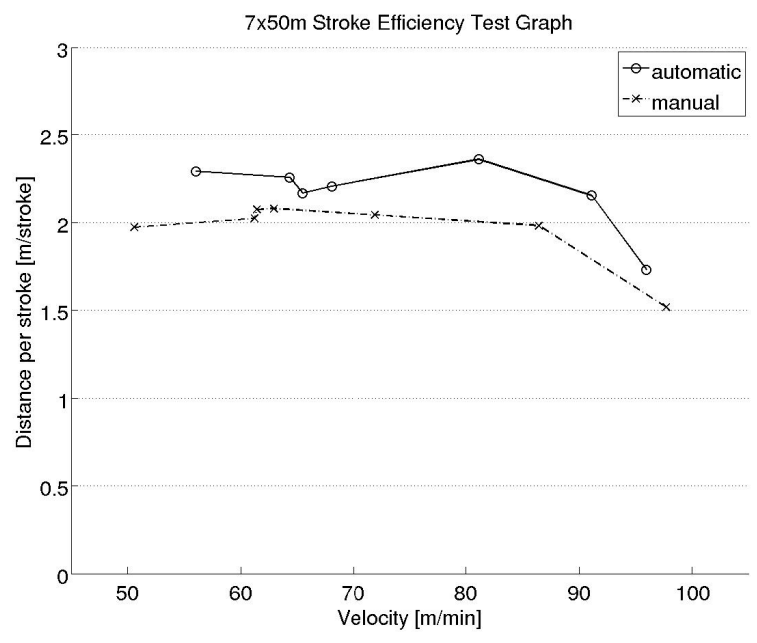

Figure 4. Swim Technique Analysis Graph measured manually and automatically

one is automatically extracted from the acceleration signal based on the whole $50 \mathrm{~m}$ lap. A comparison of both graphs shows that they have comparable trends. They differ by an offset caused by the different reference distances used. The average velocity over the $50 \mathrm{~m}$ is faster than the velocity over the $40 \mathrm{~m}$ due to the fast part after the wall push-off. Therefore the automatic measured values are at higher values on the abscissa $(V)$ and the ordinate $(D P S=V \times T P S)$.

\section{Conclusion}

We developed an automatic method to extracat the parameters of the $7 \times 50 \mathrm{~m}$ Stroke Efficiency Test based on a wrist worn acceleration sensor device. We validated the approach in a trial with eight subjects, one half from a competitive swimming club and the second half recreational swimmers. We have shown the capabilities of the automatic identification of the push-off phase, the strokes and the wall-strike by the characteristics of the swimmers' wrist acceleration. Therefore we can automatically extract the parameters for the Stroke Efficiency Test which were so far determined by the swim trainer.

We have analyzed the maximum errors from the automatic event detection with respect to the visual inspection of the signal. We have shown that the accuracy of our system is of comparable order to the manual measurement by a second person. The largest error in the automatic parameter extraction, with respect to the manual measurement, is due to the fact that the veloc- ity of the whole $50 \mathrm{~m}$ is measured instead of the pure mid-pool area. For improving the velocity measurement one possibility could be to determine the duration and/or strength of the push-off phase from the acceleration signal. With this information the error induced by the start phase could be compensated.

By implementing the described methods directly on the recording device we will be able to obtain the velocity and stroke length online. This enables a direct user feedback rather than only a later briefing by the trainer after the training session. It is further possible to store the results on the device and transmit them to a PDA or personal computer for a comparison with other swimmers or former tests. An extensive usability study has to show if such a system is accepted by the swimmers and trainers.

The automatic extraction of swim parameters saves time, works independently from a coach and therefore allows a more frequent analysis. While the traditional data collection methods are labor and technology intensive (under water cameras), a wrist watch like device offers the potential to be distributed to all swimmers for all training sessions. Furthermore, it enables the large group of recreational swimmers to evaluate their performance using techniques so far only available to competitive swimmers.

Extended research has to show if the recorded acceleration signal can also be used for evaluating further swim parameters like stroke phases or stroke phase velocity changes. One could also think of placing additional sensors on the second wrist and/or on the ankles. This could increase the robustness of the system and would allow the extraction of additional parameters.

\section{Acknowledgments}

This work was funded by the EU project DAPHNet (www.daphnet.eu), contact number: 018474-2. All views here reflect the authors' opinion and not that of the commission.

The authors would like to thank the swimmers and trainers of the SCAP (Schwimmclub Appenzell) for taking part in the trial and their outstanding support.

\section{References}

[1] AB. Jr. Craig and DR. Pendergast, "Relationships of stroke rate, distance per stroke, and velocity in competitive swimming," Med Sci Sports, vol. 11, no. 3, pp. 278-83, 1979.

[2] L. Seifert, D. Chollet, and A. Rouard, "Swimming constraints and arm coordination," Human Move- 
ment Science, vol. 26, no. 1, pp. 68-86, February 2007.

[3] L. Seifert, L. Boulesteix, D. Chollet, and J.P. Vilas-Boas, "Differences in spatial-temporal parameters and armleg coordination in butterfly stroke as a function of race pace, skill and gender," Human Movement Science, vol. 27, no. 1, pp. 96-111, February 2008.

[4] M. Beetz, B. Kirchlechner, and M. Lames, "Computerized real-time analysis of football games," IEEE Pervasive Computing, vol. 4, no. 3, pp. 33$39,2005$.

[5] M. Beetz, N. v. Hoyningen-Huene, J. Bandouch, B. Kirchlechner, S. Gedikli, and A. Maldonado, "Camera-based observation of football games for analyzing multi-agent activities," in AAMAS '06: Proceedings of the fifth international joint conference on Autonomous agents and multiagent systems, New York, NY, USA, 2006, pp. 42-49, ACM.

[6] F. Michahelles and B. Schiele, "Sensing and monitoring professional skiers," IEEE Pervasive Computing, vol. 4, no. 3, pp. 40-46, 2005.

[7] B. Auvinet, E. Gloria, G. Renault, and E. Barrey, "Runner's stride analysis: comparison of kinematic and kinetic analyses under field conditions," Science \& Sports 2002, vol. 17, pp. 92-4, 2002.

[8] J. Hey and S. Carter, "Pervasive computing in sports training," Pervasive Computing, IEEE, vol. 4, no. 3, pp. 54-, July-Sept. 2005.

[9] R. Anderson, A. Harrison, and G. M. Lyons, "Accelerometry-based feedback - can it improve movement consistency and performance in rowing?," Sports Biomechanics, vol. 4, no. 2, pp. 179$195,2005$.

[10] Y. Ohgi, "Microcomputer-based acceleration sensor device for sports biomechanics - stroke evaluation by using swimmer's wrist acceleration," Sensors, 2002. Proceedings of IEEE, vol. 1, pp. 699$704,2002$.

[11] N. P. Davey, M. E. Anderson, and D. A. James, "An accelerometer-based system for elite athlete swimming performance analysis," in Smart Structures, Devices, and Systems II. Edited by AlSarawi, Said F. Proceedings of the SPIE, Volume 5649, pp. 409-415 (2005)., S. F. Al-Sarawi, Ed., Feb. 2005, vol. 5649 of Presented at the Society of
Photo-Optical Instrumentation Engineers (SPIE) Conference, pp. 409-415.

[12] D.A. James, N. Davey, and T. Rice, "An accelerometer based sensor platform for insitu elite athlete performance analysis," Sensors, 2004. Proceedings of IEEE, pp. 1373-1376 vol.3, 24-27 Oct. 2004.

[13] G. J. Maw and S. Volkers, "Measurement and application of stroke dynamics during training in your own pool," Australian Swim. Coach, vol. 12, no. 3, pp. 34-38, 1996.

[14] M. Bächlin, D. Roggen, and G. Tröster, "Contextaware platform for long-term life style management and medical signal analysis," in Proceeding of the 2 nd Sensation International Conference, Chania, Greece, 2007.

[15] D. Bannach, K. Kunze, P. Lukowicz, and O. Amft, "Distributed modular toolbox for multi-modal context recognition," in ARCS 2006: Proceedings of the 19th International Conference on Architecture of Computing Systems., March 2006, pp. 99 113. 